\title{
Linkage network of biserial queues with a multistage flowshop scheduling in fuzzy environment
}

\author{
$\underline{\text { Sameer Sharma }}^{a}$, Deepak Gupta ${ }^{b}$ and Seema Sharma ${ }^{a}$ \\ ${ }^{a}$ Department of Mathematics, D.A.V. College, Jalandhar City, Punjab. India \\ ${ }^{b}$ Department of Mathematics, M.M. University, Mullana, Ambala, India \\ Email: samsharma31@gmail.com
}

\begin{abstract}
This paper addresses the study of a linkage network of biserial queues linked to a common server with a multistage flowshop scheduling system having ' $m$ ' machines or servers in series under fuzzy environment. Waiting lines or queues are commonly occurred both in everyday life and in a variety of business and industrial situations. Also, the flowshop scheduling is an important task when a number of jobs are to be processed through a series of machines or servers in an order. It is usually assumed that the various arrival rates, service rates and processing times of jobs/tasks are exact. However, the real world is complex and the complexity is due to the uncertainty. To express this uncertainty, the concept of fuzziness is introduced in the queuing-scheduling linkage model. Fuzzy sets theory provides a mathematical way to represent uncertainty, vagueness and fuzziness in humanistic systems. If the various arrival and service rates are expressed by possibility rather than probability, the fuzzy queuing methods would be more realistic than classical queuing theory methods. The $\alpha$-cut approach and various fuzzy arithmetic operations are used to estimate the uncertainty associated with the input parameters. The objective of the paper is to develop an algorithm to optimize the mean queue length, average waiting time and total elapsed time for the proposed queue-scheduling linkage model in fuzzy environment. The efficiency of the proposed algorithm is tested by a numerical illustration.
\end{abstract}

Keywords: Biserial queues, arrival rate, service rate, waiting time, mean queue length, processing time, completion time, fuzzy number 


\section{INTRODUCTION}

Queuing theory is one of the classical mathematical tools for studying various queue characteristics such as average waiting time and average number of customers in system. The majority of queuing optimization problems is static where the system characteristics would not change over time. Static optimization cannot meet the demand for dynamic allocation of customers or resources in complex manufacturing or communication network. In this paper a linkage model between the networks of biserial queues connected to a common server with a multistage flowshop scheduling system involving ' $m$ ' machines/servers in which the arrival rate, service rate, various possibilities and processing time of various jobs/tasks are fuzzy in nature and represented by fuzzy triangular membership functions. $\alpha$ - cut approach and fuzzy arithmetic operations are used to derive various system characteristics. The completion time of jobs/tasks in network of queues (Phase I of service) will be the setup time for first machine in flowshop scheduling of machines (Phase II of service).The proposed model finds its applications in business administration and management, computer and communication systems, traffic management, manufacturing industry, supermarkets, hospital management and in many engineering applications.

The rest of the paper is organised as follows: Section 2 reviews the in context with proposed model. Section 3 deals with fuzzy set theory to represent the uncertainty involved and various fuzzy arithmetic operations are defined. Section 4 introduces the fuzzy biserial queuing-scheduling linkage model. Section 5 deals with the solution methodology used to find the various characteristic values. Section 6 describes the algorithm proposed for optimizing various characteristics. Section 7 tests the efficiency of the proposed algorithm by a numerical illustration. The paper is concluded in Section 8 followed by the references.

\section{LITERATURE REVIEW}

Queuing theory has its beginning in the research work of a Danish engineer Erlang, A. K., when Erlang (1909) applied this theory extensively to study the behaviour of telephone networks. Jacksons (1954) studied the behaviour of a queue system containing phase type service. Reich (1957) discussed the waiting times when queues are in tandem. Suzuki (1963) studied the behaviour of system when two queues are in series. Prabhu (1967) discussed the transient behaviour of a tandem queues Singh et al (2005) discussed the steady state behaviour of a queue model comprised of two systems with biserial channels linked with a common channel. Zadeh(1978) discussed the fuzzy set as a basis for a theory of possibility. Prade (1980) applied fuzzy or possiblistic models for queuing system. Negi and Lee (1992) proposed a procedure using $\alpha$ cuts and two variable simulations to analyse fuzzy queues. The fuzzy queue model is also used by many other researchers like Kao et al (1999), Buckley et al (2001), Ke and Lin (2006), Lin et al (2007) and Sharma et al (2013). Also, one of the earliest results in flowshop scheduling is an algorithm given by Johnson (1954) for scheduling jobs on two or three machines. Campell et al (1970) gave a heuristic algorithm for ' $n$ ' jobs, ' $m$ ' machines sequencing problems. Szware (1983) studied the flowshop problem with mean completion time criterion. Maggu and Das (1985) discussed the various concepts with different constraints for two, three and multistage flowshop scheduling. Only some efforts have been made to establish a linkage between these two fields of optimization. Kumar et al (2007) discussed the concept of linkage of queues in semi-series to a flowshop scheduling system. Singh et al (2008) studied the linkage scheduling system with a serial queue network. Singh et al (2009) established the linkage of a scheduling system with biserial queue network. Gupta et al (2012, 2012a, 2012b, 2013) studied the linkage of a parallel biserial queue network with a flowshop scheduling model with an effort to optimize the total flow time, waiting time and service time In the present paper a linkage model between networks of queues consisting of a biserial server linked to a common server in series to a multi machine flowshop scheduling in fuzzy environment is considered.

\section{FUZZY SET THEORY}

Decision making is the most important scientific, social and economic endeavour. To be able to make consistent and correct choice is the essence of any decision process imbued with uncertainty. In real world, there is some information such as the arrival rate of customers in the system or service rate and processing time of jobs/tasks can be expressed with linguistic data and it cannot be suitably expressed with probability distribution. The fuzzy logic is a tool to consider these uncertainties or vagueness. 


\subsection{Definition}

A cover and normalized fuzzy set defined on $\mathrm{R}$ whose membership function is piecewise continuous is called a fuzzy number. It is simply an ordinary number whose precise value is somewhat uncertain. A triangular fuzzy number $\tilde{\mathrm{A}}$ with membership function $\mu_{A}(X)$ defined on $\mathrm{R}$ by

$\tilde{\mathrm{A}}=\left\{\begin{array}{cl}\frac{x-a_{1}}{a_{m}-a_{1}} & , a_{1} \leq x \leq a_{m} \\ \frac{a_{2}-x}{a_{2}-a_{m}} & , a_{m} \leq x \leq a_{2}, \\ 0 & , \text { Otherwise }\end{array}\right.$

Where, the points $a_{m} \in\left(a_{1}, a_{2}\right)$ are located at the mid of the supporting interval, .i.e. three values $a_{1}, a_{m}$ and $a_{2}$ represent a triangular number denoted by $\tilde{\mathrm{A}}=\left(a_{1}, a_{m}, a_{2}\right)$. For a fuzzy set $\tilde{\mathrm{A}}$ defined on set $\mathrm{X}$ of reals, for any $\alpha \in[0,1]$, the $\alpha$-cut ${ }^{\alpha} \mathrm{A}$ is a crisp set that contains all the elements of $\mathrm{X}$ that have membership value greater than or equal to $\alpha$.

\subsection{Fuzzy Arithmetic Operations}

Let $\tilde{\mathrm{A}}=\left(a_{1}, a_{2}, a_{3}\right)$ and $\tilde{\mathrm{B}}=\left(b_{1}, b_{2}, b_{3}\right)$ be the two triangular fuzzy numbers, then the four basic operations that can be performed on triangular fuzzy numbers are as follows:

1. Addition: $\tilde{A}+\tilde{B}=\left(a_{1}+b_{1}, a_{2}+b_{2}, a_{3}+b_{3}\right)$.

2. Subtraction: $\tilde{\mathrm{A}}-\tilde{\mathrm{B}}=\left(a_{1}-b_{1}, a_{2}-b_{2}, a_{3}-b_{3}\right)$.

This subtraction operation exist only if the following condition is satisfied $\mathrm{DP}(\mathrm{A}) \geq \mathrm{DP}(\mathrm{B})$, where $D P(\tilde{\mathrm{A}})=\left(a_{3}-a_{1}\right) / 2$ and $D P(\tilde{\mathrm{B}})=\left(b_{3}-b_{1}\right) / 2$; else, $\tilde{\mathrm{A}}-\tilde{\mathrm{B}}=\left(a_{1}-b_{3}, a_{2}-b_{2}, a_{3}-b_{1}\right)$.

3. Multiplication: $\tilde{\mathrm{A}} \times \tilde{\mathrm{B}}=\left(a_{1} b_{2}+a_{2} b_{1}-a_{2} b_{2}, a_{2} b_{2}, a_{2} b_{3}+a_{3} b_{2}-a_{2} b_{2}\right)$.

4. Division: $\tilde{\mathrm{A}} / \tilde{\mathrm{B}}=\left(\frac{2 a_{1}}{b_{1}+b_{3}}, \frac{a_{2}}{b_{2}}, \frac{2 a_{3}}{b_{1}+b_{3}}\right)$; provided that $\tilde{\mathrm{A}}$ and $\tilde{\mathrm{B}}$ are all non zero numbers.

\section{FUZZY BISERIAL QUEUING-SCHEDULING LINKAGE MODEL}

According to Zadeh development theory (1978) and by the use of the possibility theory concepts, fuzzy Markov chain (1982), Li and Lee (1989) proposed that every fuzzy queuing model could be considered as a classical queuing theory model by considering following changes: the probability distribution function of the time between two consecutive arrivals which is assumed to follow the exponential distribution with the parameter $\lambda$ being considered to be $\tilde{\lambda}$ in fuzzy environment which is an approximation of the mean of its possibility distribution. Similarly, the servicing rate $\mu$ is considered to be the fuzzy number $\tilde{\mu}$ which is the mean of its possibility distribution in fuzzy environment. The following notations have been used throughout the course of the present research paper:

$\lambda$ : fuzzy arrival rate; $\mu$ : fuzzy service rate; $n$ : Number of jobs / tasks arriving; $\tilde{P}_{i j}(s)$ : possibility that the system is in the state $(i, j)$ at any time $\mathrm{t} ; L:$ mean queue length of the jobs/tasks in system; $E(W)$ :expected waiting time jobs/tasks; $\tilde{L}_{S}$ : fuzzy mean queue length (number of jobs) of the system; $E\left(\tilde{W}_{S}\right)$ : expected fuzzy waiting time of the system; $\tilde{A}_{i, j}$ : fuzzy processing time of $i^{\text {th }}$ job on $j^{\text {th }}$ machine or server; $A_{i, j}$ : AHR of fuzzy processing time of $i^{\text {th }}$ job on $j^{\text {th }}$ machine or server.

The proposed fuzzy biserial queuing-scheduling linkage model has two service phases. The phase I of service includes the fuzzy biserial queue model comprised of two servers $S$ and $S^{\prime}$. The server $S$ consists of two biserial service servers $S_{1}$ and $S_{2}$. The service server $S^{\prime}$ is commonly linked in series with each of the two parallel biserial servers $S_{1}$ and $S_{2}$. Let $n_{1}, n_{2}$ be the number of customers arriving at the servers $S_{1}$ and $S_{2}$ with fuzzy arrival rates $\tilde{\lambda}_{1}$ and $\tilde{\lambda}_{2}$. Let $\mu_{1}$ and $\mu_{2}$ be the fuzzy service rates at servers $S_{1}$ and $S_{2}$ and $\mu_{3}$ be at 
server $S^{\prime}$. Queues $Q_{1}, Q_{2}, Q_{3}$ are said to be formed in front of the service servers $S_{1}, S_{2}$ and server $S^{\prime}$, if they are busy. Customers or jobs arriving at rate $\lambda_{1}$ after completion of phase service at $S_{1}$ will join $S_{2}$ or $S^{\prime}$ i.e., either they may follow the network of servers $S_{1} \rightarrow S_{2} \rightarrow S^{\prime}$ or $S_{1} \rightarrow S^{\prime}$ with possibilities $P_{12}$ or $P_{13}$ such that $\tilde{P}_{12}+P_{13}=1$. Also, the customers or jobs at rate $\lambda_{2}$ after completion of phase service at $S_{2}$ will join $S_{\downarrow}$ or $S^{\prime}$ i.e., either they may follow the network of servers $S_{2} \rightarrow S_{1} \rightarrow S^{\prime}$ or $S_{2} \rightarrow S^{\prime}$ with possibilities $P_{21}$ or $P_{23}$ such that $P_{21}+P_{23}=1$. The phase II of service has a system of servers (machines) in series. After completing the phase I of service, various jobs/tasks have to be processed through system of machines with fuzzy processing time $A_{i, j}$. Further the completion time of jobs/tasks in phase I of service will be the set up time for first machine in phase II of service.

In this model, the arrival rate $\tilde{\lambda}$, the service rate $\tilde{\mu}$ and the processing time of jobs on various machines $\tilde{A}_{i, j}$ are approximately known and can be represented as convex fuzzy sets. Let $\eta_{\tilde{\lambda}}(\tilde{\lambda}), \eta_{\tilde{\mu}}(\tilde{\mu})$ and $\eta_{\tilde{A_{i, j}}}\left(\tilde{A_{i, j}}\right)$ denote the membership functions of $\tilde{\lambda}, \tilde{\mu}$ and $\tilde{A_{i, j}}$ respectively. The fuzzy sets are described as

$\tilde{\lambda}=\left\{\left(\lambda, \eta_{\tilde{\lambda}}(\tilde{\lambda})\right) / \lambda \in X\right\}, \tilde{\mu}=\left\{\left(\mu, \eta_{\tilde{\mu}}(\tilde{\mu})\right) / \mu \in Y\right\}, \tilde{A_{i, j}}=\left\{\left(A_{i, j}, \eta_{\tilde{A}_{i, j}}\left(\tilde{A_{i, j}}\right)\right) / A_{i, j} \in Z\right\}$

where $\mathrm{X}, \mathrm{Y}$ and $\mathrm{Z}$ are the crisp universal sets of arrival rates and service rates respectively.

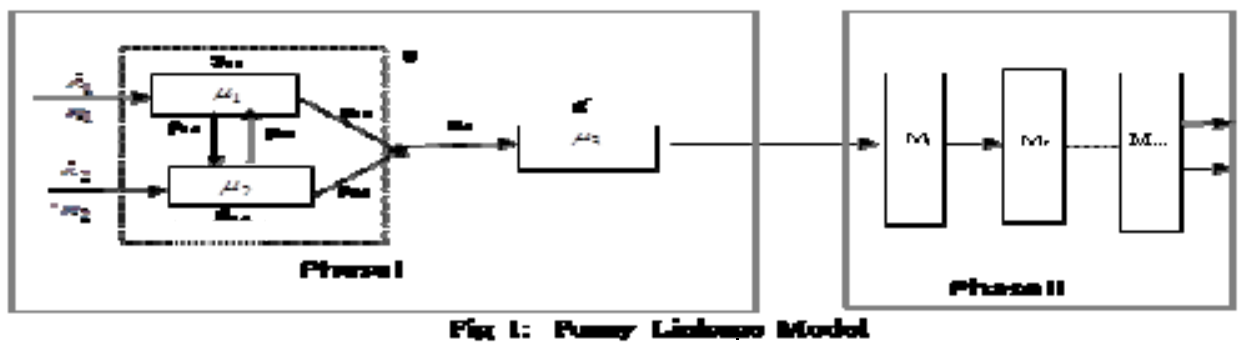

\section{SOLUTION METHODOLOGY}

The system data which are gathered from the real world always has a sort of ambiguity. The "fuzzy set" is a tool to consider these ambiguities. On the other hand, to determine the parameters of the model in the real world, generally, the expert ideas or sampling data can be used. It can be claimed that there is a sort of ambiguity in the both mentioned methods. In the first case, the ambiguity is due to the lack of the preciseness and enough specialties. In the second case, the ambiguity is due to the lack of enough sampling data. Because of this ambiguity, in this paper, it is preferred to use the fuzzy parameters for the suggested queuing-scheduling linkage model. To estimate a parameter as a fuzzy number and to change the input data (Arrival rate, Service rate and Processing time) into the fuzzy numbers, the method of Buckley and Qu (1990) is used.

Since the arrival rate $(\tilde{\lambda})$, the service rate $(\tilde{\mu})$ and the processing time $\left(\tilde{A}_{i, j}\right)$ are not known precisely, we use triangular fuzzy numbers to represent them. The fuzzy arrival rates $\tilde{\lambda}_{1}, \tilde{\lambda}_{2}$; the fuzzy service rates $\tilde{\mu_{1}}, \tilde{\mu_{2}}, \tilde{\mu_{3}}$, various fuzzy possibilities $\tilde{p_{12}}, \tilde{p_{13}}, \tilde{p_{21}}, \tilde{p_{23}}$ and the fuzzy processing times $\tilde{A_{i, j}}$ be defined as $\tilde{\lambda}_{i}=\left(\lambda_{i}^{1}, \lambda_{i}^{2}, \lambda_{i}^{3}\right), \tilde{\mu}_{i}=\left(\mu_{i}^{1}, \mu_{i}^{2}, \mu_{i}^{3}\right), \tilde{p}_{i j}=\left(p_{i j}^{1}, p_{i j}^{2}, p_{i j}^{3}\right)$ and $\tilde{A}_{i j}=\left(A_{i j}^{1}, A_{i j}^{2}, A_{i j}^{3}\right)$ for various values of $i$ and $j$, where $\lambda_{i}^{1}<\lambda_{i}^{2}<\lambda_{i}^{3}, \mu_{i}^{1}<\mu_{i}^{2}<\mu_{i}^{3}$ and $A_{i, j}^{1}<A^{2}{ }_{i, j}<A^{3}{ }_{i, j}$ based on subjective judgment.

Using the concept of $\alpha$ - cut method, we have

$$
\begin{aligned}
& { }^{\alpha} \lambda_{i}=\left[\alpha\left(\lambda_{i}^{2}-\lambda_{i}^{1}\right)+\lambda_{i}^{1}, \lambda_{i}^{3}-\alpha\left(\lambda_{i}^{3}-\lambda_{i}^{2}\right)\right],{ }^{\alpha} \mu_{i}=\left[\alpha\left(\mu_{i}^{2}-\mu_{i}^{1}\right)+\mu_{i}^{1}, \mu_{i}^{3}-\alpha\left(\mu_{i}^{3}-\mu_{i}^{2}\right)\right], \\
& { }^{\alpha} p_{i j}=\left[\alpha\left(p_{i j}^{2}-p_{i j}^{1}\right)+p_{i j}^{1}, p_{i j}^{3}-\alpha\left(p_{i j}^{3}-p_{i j}^{2}\right)\right] \text { and }{ }^{\alpha} A_{i j}=\left[\alpha\left(A_{i j}^{2}-A_{i j}^{1}\right)+A_{i j}^{1}, A_{i j}^{3}-\alpha\left(A_{i j}^{3}-A_{i j}^{2}\right)\right] .
\end{aligned}
$$


The system characteristic of interest are the average queue length, the expected waiting time and the total elapsed time of jobs / tasks. From the previous knowledge of queue theory as given by Sharma et al (2013), we have, the average (mean) fuzzy queue length for the biserial queue model under consideration is

$$
\tilde{L}=\tilde{L}_{1}+\tilde{L}_{2}+\tilde{L}_{3}=\left(L_{1}^{1}, L_{1}^{2}, L_{1}^{3}\right)+\left(L_{2}^{1}, L_{2}^{2}, L_{2}^{3}\right)+\left(L_{3}^{1}, L_{3}^{2}, L_{3}^{3}\right)=\left(L_{1}^{1}+L_{2}^{1}+L_{3}^{1}, L_{3}^{2}+L_{3}^{2}+L_{3}^{2}, L_{1}^{3}+L_{2}^{3}+L_{3}^{3}\right)=\left(l_{1}, l_{2}, l_{3}\right)
$$

where, $\tilde{L}_{1}=\left(\frac{\lambda_{1}^{1}+\lambda_{2}^{1} p_{21}^{1}}{\left(1-p_{12}^{3} p_{21}^{3}\right) \mu_{1}^{3}}, \frac{\lambda_{1}^{3}+\lambda_{2}^{3} p_{21}^{3}}{\left(1-p_{12}^{1} p_{21}^{1}\right) \mu_{1}^{2}}, \frac{\lambda_{1}^{2}+\lambda_{2}^{2} p_{21}^{2}}{\left(1-p_{12}^{2} p_{21}^{2}\right) \mu_{1}^{2}}\right)=\left(L_{1}^{1}, L_{1}^{2}, L_{1}^{3}\right)$,

$$
\begin{gathered}
\tilde{L}_{2}=\left(\frac{\lambda_{2}^{1}+\lambda_{1}^{1} p_{12}^{1}}{\left(1-p_{12}^{3} p_{21}^{3}\right) \mu_{2}^{3}}, \frac{\lambda_{2}^{3}+\lambda_{1}^{3} p_{12}^{3}}{\left(1-p_{12}^{1} p_{21}^{1}\right) \mu_{2}^{2}}, \frac{\lambda_{2}^{2}+\lambda_{1}^{2} p_{12}^{2}}{\left(1-p_{12}^{2} p_{21}^{2}\right) \mu_{2}^{2}}\right)=\left(L_{2}^{1}, L_{2}^{2}, L_{2}^{3}\right), \\
\tilde{L}_{3}=\left(\frac{p_{13}^{1}\left(\lambda_{1}^{1}+\lambda_{2}^{1} p_{21}^{1}\right)+p_{23}^{1}\left(\lambda_{2}^{1}+\lambda_{1}^{1} p_{12}^{1}\right)}{\left(1-p_{12}^{1} p_{21}^{1}\right) \mu_{3}^{3}}, \frac{p_{13}^{3}\left(\lambda_{1}^{3}+\lambda_{2}^{3} p_{21}^{3}\right)+p_{23}^{3}\left(\lambda_{2}^{3}+\lambda_{1}^{3} p_{12}^{3}\right)}{\left(1-p_{12}^{3} p_{21}^{3}\right) \mu_{3}^{2}}, \frac{p_{13}^{2}\left(\lambda_{1}^{2}+\lambda_{2}^{2} p_{21}^{2}\right)+p_{23}^{2}\left(\lambda_{2}^{2}+\lambda_{1}^{2} p_{12}^{2}\right)}{\left(1-p_{12}^{2} p_{21}^{2}\right) \mu_{3}^{2}}\right)=\left(L_{3}^{1}, L_{3}^{2}, L_{3}^{3}\right),
\end{gathered}
$$$$
\tilde{\lambda}=\tilde{\lambda}_{1}+\tilde{\lambda}_{2}=\left(\lambda_{1}^{1}, \lambda_{1}^{2}, \lambda_{1}^{3}\right)+\left(\lambda_{2}^{1}, \lambda_{2}^{2}, \lambda_{2}^{3}\right)=\left(\lambda_{1}^{1}+\lambda_{2}^{1}, \lambda_{1}^{2}+\lambda_{2}^{2}, \lambda_{1}^{3}+\lambda_{2}^{3}\right)=\left(\delta_{1}, \delta_{2}, \delta_{3}\right)
$$

Also, the fuzzy average waiting time of the customers / jobs is $E(\tilde{W})=\frac{\tilde{L}}{\tilde{\lambda}}=\frac{\left(l_{1}, l_{2}, l_{3}\right)}{\left(\delta_{1}, \delta_{2}, \delta_{3}\right)}=\left(w_{1}, w_{2}, w_{3}\right)$

Further, the total fuzzy completion time $(\tilde{C})$ of jobs/tasks for the phase I of service is:

$$
\tilde{C}=E(\tilde{W})+\frac{1}{\tilde{\mu}_{1} \tilde{p}_{12}+\tilde{\mu}_{1} \tilde{p}_{13}+\tilde{\mu}_{2} \tilde{p_{21}}+\tilde{\mu}_{2} \tilde{p}_{23}+\tilde{\mu}_{3}}=\left(c_{1}, c_{2}, c_{3}\right)
$$

The total fuzzy completion time will be the setup time for the first machine in Phase II of service. To find the optimal sequence of jobs/tasks in order to minimize the total elapsed time when a set of ' $n$ ' jobs entered into the phase II of service consisting of ' $m$ ' machines in series with no passing and pre-emission being allowed, the following result by Sharma et al (2013) is considered:

Statement: Assume $n$ jobs $J_{l}, J_{2}, J_{3} \ldots J_{n}$ are processed through ' $m$ ' machines $M_{j}(j=1,2 \ldots \mathrm{m})$ in order $M_{l^{-}}$ $M_{2}-M_{3}-\ldots \ldots-M_{m}$ with no passing allowed. If $A_{i, j}$ represents the crisp values of fuzzy processing time $\tilde{A}_{i, j}$ of $i^{\text {th }}$ job $(i=1,2, \ldots, \mathrm{n})$ on $j^{\text {th }}$ machine $(j=1,2, \ldots, \mathrm{m})$ such that $\min A_{i, s} \geq \max A_{i,(s+1)} ; s=1,2, \ldots,(m-2)$, then the optimal schedule minimizing the total elapsed time is given by the following decision rule: Job $J_{k}$ proceeds job $J_{k+1}$ if $\min \left\{G_{k}, H_{k+1}\right\}<\min \left\{G_{k+1}, H_{k}\right\}$; where $G_{i}=A_{i, 1}+A_{i, 2}+---+A_{i,(m-1)}$ and $H_{i}=A_{i, 2}+A_{i, 3}$ $+---+A_{i, m}$.

Since the system characteristics are described by membership function, it preserves the fuzziness of input information. However, the designer would prefer one crisp value for one of the system characteristics rather than the fuzzy set. In order to overcome this problem we defuzzify the fuzzy system characteristic values by using the Yager's (1981) formula: if $\tilde{A}=\left(a_{1}, a_{2}, a_{3}\right)$ is a fuzzy number, then crisp $A=\frac{a_{1}+2 a_{2}+a_{3}}{4}$.

\section{PROPOSED ALGORITHM}

The following algorithm gives the procedure to determine the optimal sequence of jobs/tasks for the linkage network of biserial queues with the scheduling of jobs in a multistage flowshop setup having ' $m$ ' machines in series under fuzzy environment. The various characteristics, e.g., mean queue length, total waiting time of jobs/customers, total completion time and minimum total flow time of jobs/customers for the proposed linkage model are established. The various steps of the proposed algorithm are as follows:

Step 1: Find the mean queue length according to Sharma et al (2013) using the formula:

$$
\tilde{L}=\tilde{L}_{1}+\tilde{L}_{2}+\tilde{L}_{3}=\left(L_{1}^{1}, L_{1}^{2}, L_{1}^{3}\right)+\left(L_{2}^{1}, L_{2}^{2}, L_{2}^{3}\right)+\left(L_{3}^{1}, L_{3}^{2}, L_{3}^{3}\right)=\left(L_{1}^{1}+L_{2}^{1}+L_{3}^{1}, L_{3}^{2}+L_{3}^{2}+L_{3}^{2}, L_{1}^{3}+L_{2}^{3}+L_{3}^{3}\right)=\left(l_{1}, l_{2}, l_{3}\right)
$$

Step 2: Find the average fuzzy waiting time $E(\tilde{W})$ of the customers. 
Sharma, S. et al., Linkage network of biserial queues in fuzzy environment

Step 3: Find the total fuzzy completion time of jobs/customers coming out of Phase I of the service, .i.e. when processed through the network of queues.

Step 4: The total fuzzy completion time will be the setup time for the first machine in Phase II of the service.

Define the machines $M_{1}, M_{2} \ldots, M_{m}$ with processing time $\tilde{A}_{i, 1}=\tilde{A}_{i, 1}+C$ and $\tilde{A}_{i, 2}, \tilde{A}_{i, 3}, \ldots, \tilde{A_{i, m}}$.

Step 5: Check the consistency of the structural conditions so as to reduce ' $n$ ' jobs ' $m$ ' machines problem to ' $n$ ' jobs 2 machine problem. If holds, then define ' $n$ ' jobs, 2-machine problem as follows:

Introduce the two fictitious machines $G$ and $H$ with processing time $G_{i}$ and $H_{i}$ defined by

$G_{i}=\tilde{A}_{i, 1}+\tilde{A}_{i, 2}+------+\tilde{A}_{i,(m-1)}^{\sim}$ and $H_{i}=\tilde{A}_{i, 2}+\tilde{A}_{i, 3}+------+\tilde{A}_{i, m}$.

Step 6: Apply modified technique as established by Sharma et al (2013) to find the optimal sequence(s) of jobs with minimum total elapsed time.

Step 7: Prepare In-out table for the sequence of jobs as in step 6. The sequence $S$ having minimum total elapsed time will be the optimal sequence for the given linkage model with mean queue length $\tilde{L}$ and $E(\tilde{W})$ as average waiting time of jobs/tasks in queuing- scheduling linkage model.

\section{TEST OF PROPOSED ALGORITHM}

Consider seven jobs/tasks processed through the network of queues $Q_{1}, Q_{2}$ and $Q_{3}$ with the servers $S_{1}, S_{2}$ and $S^{\prime}$. The servers $S_{1}$ and $S_{2}$ are parallel biserial servers and server $S^{\prime}$ linked in series with each of two servers $S_{1}$ and $S_{2}$. The number of the customers, fuzzy mean arrival rate, fuzzy mean service rate and various associated possibilities are given in Table 1.

Table 1: Input variable of the proposed biserial queue model

\begin{tabular}{|c|l|l|l|l|}
\hline S.No. & $\begin{array}{l}\text { No. of } \\
\text { Customers }\end{array}$ & Arrival Rate & Service Rate & Possibilities \\
\hline 1 & $\mathrm{n}_{1}=3$ & $\tilde{\lambda}_{1}=(5,6,7)$ & $\tilde{\mu}_{1}=(12,14,16)$ & $\tilde{p_{12}}=(0.6,0.7,0.8)$ \\
\hline 2 & $\mathrm{n}_{2}=4$ & $\tilde{\lambda}_{2}=(4,5,6)$ & $\tilde{\mu}_{2}=(14,15,16)$ & $\tilde{p_{13}}=(0.4,0.3,0.2)$ \\
\hline & & & $\tilde{\mu}_{3}=(9,12,15)$ & $\tilde{p_{21}}=(0.3,0.5,0.6)$ \\
\hline & & & & $\sim$ \\
& & & & $\tilde{p}_{23}=(0.7,0.5,0.4)$ \\
\hline
\end{tabular}

Here, we have $\tilde{L}_{1}=(0.7452,0.9233,0.9341), \tilde{L}_{2}=(0.8413,0.9431,0.9436)$ and $\tilde{L}_{3}=(0.9461,0.6867,0.7542)$.

Therefore, the mean (average) queue length of jobs/tasks $\tilde{L}=\tilde{L}_{1}+\tilde{L}_{2}+\tilde{L}_{3}=(2.5326,2.5531,2.6319)$.

It indicates that the mean queue length of customers (jobs) in the system falls between 2.5326 and 2.6319,

The expected waiting time of the jobs/tasks $E(\tilde{W})=(0.2302,0.2321,0.2393)$ ranges from 0.2302 to 0.2393 .

On defuzzifing, we get $E(L)=2.567675, E(W)=0.233425$

Also, the total fuzzy completion time of jobs/tasks in getting phase I of service is $\tilde{C}=(0.2531,0.2563,0.2637)$. After being served in phase I, the various jobs/tasks entered in phase II of service have a system of three machines/servers in series. The fuzzy processing times of various jobs on these machines are as follows:

Table 2: Jobs with processing time

\begin{tabular}{|l|l|l|l|l|l|l|l|}
\hline Jobs & 1 & 2 & 3 & 4 & 5 & 6 & 7 \\
\hline $\mathrm{M}_{1}$ & $(7,8,9)$ & $(12,13,14)$ & $(8,10,12)$ & $(10,11,12)$ & $(9,10,11)$ & $(8,10,12)$ & $(12.13 .14)$ \\
\hline $\mathrm{M}_{2}$ & $(6,7,8)$ & $(5,6,7)$ & $(4,5,6)$ & $(5,6,7)$ & $(6,7,8)$ & $(4,5,6)$ & $(5,6,7)$ \\
\hline $\mathrm{M}_{3}$ & $(3,4,5)$ & $(4,5,6)$ & $(6,7,8)$ & $(11,12,13)$ & $(8,9,10)$ & $(6,7,8)$ & $(4,5,6)$ \\
\hline
\end{tabular}

On using various steps, the optimal sequence of jobs is $S=4-5-6-3-7-2-1$ and the total elapsed time of the jobs comes to be $(76.7717,77.7961,93.8196)$. It indicates that the total elapsed time of jobs/tasks for the proposed queuing-scheduling model lies between 76.7717 and 93.8196 . 
Sharma, S. et al., Linkage network of biserial queues in fuzzy environment

\section{CONCLUSION}

The fuzzy systems are very useful in two general contexts: (1) in situations involving highly complex system whose behaviour are not well understood and (2), in situations where an approximate but fast solution is warranted. In this paper the fuzzy logic approach is used to represents the uncertainty or vagueness or complexity or impreciseness in the arrival rate, service rate, various possibilities and the processing time of various jobs/tasks. An algorithm is developed to find the mean queue length, average waiting time and minimum possible total elapsed time for the proposed queuing-scheduling linkage model in fuzzy environment. The study may further be extended by introducing more complex network of queues in phase I of service in fuzzy environment.

\section{REFERENCES}

Buckley, J.J. and Qu, Y. (2004). On using $\alpha$ cuts to evaluate fuzzy equations. Fuzzy Sets and Systems, 38, 309-312.

Campell, H. A., Duder, R. A. and Smith, M. L. (1970). A heuristic algorithm for n-jobs, m-machines sequencing problem. Management Science, 16, B630-B637.

Erlang, A.K. (1909). The theory of probabilities and telephone conversations. ,Nyt Tidsskrift fur Matematik, $\mathrm{B}(20), 33$.

Gupta, D., Sharma, S. and Seema (2012). On linkage of a flow shop scheduling model including job block criteria with a parallel biserial queue network. Computer Engineering and Intelligent Systems, 3(2), 17-28.

Gupta, D., Sharma, S. and Seema (2012a). On linkage of a queue network with biserial and parallel channels linked with a common server to a two stage flowshop scheduling system. International Journal of Emerging trends in Engineering and Development, 2( 2), 265-275.

Gupta, D., Sharma, S. and Seema (2012b). On linkage of parallel biserial servers linked with a common server to a three stage flowshop scheduling model. International Journal of Applied Physics and Mathematics, 2(3), 152-154.

Jackson, R. R. P. (1954). Queuing system with phase type service. O. R. Quarterly, 5, 109-120.

Johnson, S. M. (1954). Optimal two \& three stage production schedules with set up times includes. Nav. Res. Log. Quart., 1, 61-68.

Negi, D.S. and Lee, E.S. (1992). Analysis and simulation of fuzzy queues. Fuzzy Sets and Systems, 46, 321330.

Singh, T. P., Kumar, V. and Kumar, R. (2005). On transient behaviour of a queuing network with parallel biserial queues. JMASS, 1(2), 68-75.

Suzuki, T. (1963). Two queues in series. Journal of Operational Research Society of Japan, 5, 149-155.

Singh, T. P., Kumar, V. (2007). On Linkage of queues in semi-series to a flowshop scheduling system, Int. Agrkult. Stat. Sci., 3(2), 571-580.

Singh, T. P., Kumar, V. and Kumar, R. (2008). Linkage scheduling system with a serial queue-network. Lingaya's Journal of professional studies, 2(1), 25-30.

Singh, T. P., Kumar, V. (2009). On linkage of a scheduling system with biserial queue network. Arya Bhatta Journal of Mathematics \& Informatics, 1(1), 71- 76.

Sharma, S., Gupta, D. and Sharma, S. (2013). Analysis of biserial queue network in fuzzy environment. International Journal of Computer Application, 68(6), 26-30.

Sharma, S., Gupta, D. and Sharma, S. (2013) 'Analysis of linkage network of queues with a multistage flowshop scheduling system' Accepted for publication in International Journal of Operational Research.

Yager, R.R., (1981). A procedure for ordering fuzzy subsets of the unit interval, Inform. Sci., 24, 143-161.

Zadeh, L. A., (1978). Fuzzy sets as a basis for a theory of possibility. Fuzzy Sets and Systems, 1, 3-28. 